\title{
Observations sur la spécificité des Acariens de la famille Myobiidae.
}

\author{
Corrélation entre l'évolution des parasites \\ et de leurs hôtes *
}

\author{
par A. FAIN \\ Institut de Médecine Tropicale Prince-Léopold, 155, rue Nationale, B-2000 Anvers
}

\section{Résumé.}

Les Acariens sont des parasites particulièrement intéressants dans l'étude de la spécificité et de l'évolution parallèle hôte-parasite. Les groupes qui se prêtent le mieux à cette étude sont les parasites permanents, c'est-à-dire qui ne quittent leur hôte à aucun moment de leur développement. Parmi ceux-ci les Acariens pilicoles, qui s'attachent aux poils de l'hôte, présentent une spécificité particulièrement stricte.

L'auteur étudie la spécificité et la corrélation entre l'évolution des hôtes et de leurs parasites chez les Acariens pilicoles de la famille Myobiidae.

Il montre que dans cette famille c'est la stucture de la patte I, c'est-à-dire celle qui sert à la fixation sur l'hôte, qui est le meilleur témoin de l'évolution. En se basant sur ce caractère il est possible de distinguer 3 grands groupes chez les Myobiidae. Dans le premier groupe, le moins évolué, la patte I est bien développée avec tous les articles segmentés et portant apicalement une paire de griffes. Ce groupe comprend tous les genres et espèces connus chez les Marsupiaux et le genre le plus primitif connu chez les Insectivores. Dans le deuxième groupe, la patte I présente une réduction plus ou moins marquée des segments apicaux avec chez la plupart des espèces une fusion plus ou moins complète du tarse et du tibia. Les griffes terminales sont présentes ou absentes. Ce groupe est plus évolué que le précédent. Il comprend tous les genres et espèces vivant sur les Insectivores, excepté un genre qui fait partie du groupe I, et tous les genres et espèces vivant sur les Chiroptères. Dans le troisième groupe, la patte I est fortement raccourcie et présente une fusion complète

* Communication présentée au Congrès du Centenaire de la Société Zoologique de France (Paris, 5-11 septembre 1976).

Reçu le 10 novembre 1976. 
des 3 segments apicaux. Les griffes manquent toujours. Ce groupe est complètement inféodé aux Rongeurs.

L'auteur étudie la spécificité et l'évolution parallèle hôte-parasite chez les différents groupes d'hôtes parasités par les Myobiidae, c'est-à-dire Marsupiaux, Insectivores, Chiroptères et Rongeurs.

L'évolution parallèle hôtes-parasites est généralement bien marquée chez tous les ordres d'hôtes, excepté cependant pour certaines familles ou sous-familles d'Insectivores ou de Chiroptères. C'est ainsi que les Myobiidae parasitant les Tenrecidae et les Macroscelididae sont nettement plus évolués que tous les genres de Myobiidae (excepté Blarinobia) vivant sur les Soricidae et les Talpidae. Notons aussi que les Soricinae hébergent à la fois les deux genres les plus primitifs (Nectogalobia et Protomyobia) et le genre le plus évolué (Blarinobia) connus chez les Insectivores. Un phénomène semblable est observé chez les Chiroptères, où ce ne sont pas les Megachiroptères qui hébergent les Myobiidae les plus primitifs, mais bien certains Microchiroptères, comme les Vespertilionidae. Cette situation paradoxale ne peut s'expliquer que si l'on admet que certains Myobiidae sont passés secondairement d'hôtes évolués sur des hôtes plus primitifs.

Notons à cet égard que la situation inverse, à savoir la présence d'un Myobiidae primitif sur un hôte évolué n'a jamais été observée, ce qui tendrait à montrer que le parasite primitif est moins apte que le parasite évolué à s'adapter à un nouvel hôte en particulier quand ce dernier est évolué.

\section{Summary:}

Study on specificity and parallel host-parasite evolution in mites of the family Myobiidae.

Mites are very interesting parasites for the study of specificity and parallel hostparasite evolution. The groups most suitable for this study are the permanent parasites which do not leave their host at any time. Among these, the fur-mites, which attach to the hairs of the host, have a particularly strict specificity.

The author studies the specificity and the correlation between the hosts and their parasites in the fur-mites of the family Myobiidae. He shows that in this family, leg I is the best criterium of the evolution. Using this character it is possible to distinguish 3 main groups in the Myobiidae. In the first group, the less evolved, leg I is well developed with all the articles segmented and bearing a pair of claws. This group includes all the genera and species known in the Marsupialia and the most primitive genus known in the Insectivora. In the second group, the apical segments of leg $\mathrm{I}$ are reduced and tarsus and tibia I are more or less completely fused. The apical claws may be present or absent. This group is more evolved than the preceeding one. It includes all the genera and species living on Insectivora, except one genus which belongs to group I, and all the genera and species living on Chiroptera. In the third group, leg $\mathrm{I}$ is strongly reduced and the 3 apical segments are fused. The claws are always lacking. This group is specialized for Rodentia.

The author studies the specificity and the parallel host-parasite evolution in the different host groups parasitized by Myobiidae, e.g. Marsupialia, Insectivora, Chiroptera and Rodentia. 
The parallel host-parasite evolution is generally well marked in all the host orders, except in some families or subfamilies of Insectivora or Chiroptera. For example the Myobiidae living on Tenrecidae and Macroscelididae are distinctly more evolved than all thé genera of Myobiidae (except Blarinobia) living on Soricidae and Talpidae. Also it should be noted that the Soricinae harbor both the two most primitive genera (Nectogalobia and Protomyobia) and the most evolved genus (Blarinobia) known in the Insectivora. A similar situation exists in the Chiroptera where it is not the Megachiroptera which harbor the most primitive Myobiidae but some of the Microchiroptera, e.g. Vespertilionidae. This paradoxal situation can be explained only if one accepts the idea that some Myobiidae have been secondarily passed from the more evolved hosts to primitive hosts. It should be emphasized that the reverse situation, e.g. the presence of a primitive myobiid on an evolved host, has never been observed, which tends to show that a primitive parasite is less capable of adapting to a new host, especially an evolved host, than an evolved parasite.

\section{Introduction}

Lors d'un Symposium organisé à Neuchâtel en 1957 sur la spécificité parasitaire, il est clairement apparu que les parasites pouvaient fournir des indications de valeur sur la position systématique des hôtes sur lesquels ils vivent.

Les Acariens n'étaient pas représentés à ce Symposium, probablement parce que, à cette époque, ces parasites étaient encore relativement peu étudiés, et que l'on ne possédait pas de vue d'ensemble sur aucun groupe important.

Ces lacunes ont été comblées au cours de ces deux dernières décennies, et actuellement beaucoup de groupes commencent à être bien connus.

\section{Abondance et diversité de la faune des Acariens parasites}

Les Acariens comptent de très nombreuses espèces qui vivent en parasites soit à la surface du corps, soit dans les organes profonds de leurs hôtes.

Des Acariens parasites sont présents dans tous les groupes de Vertébrés, excepté cependant chez les poissons. C'est chez les Vertébrés à sang chaud, Mammifères et oiseaux, qu'ils sont le mieux représentés.

Les Vertébrés ne sont pas les seuls à héberger des Acariens. Ceux-ci sont également abcndants sur les Invertébrés, surtout les insectes.

\section{Intérêt des Acariens}

\section{dans l'étude des phénomènes liés au parasitisme}

Les Acariens parasites sont plus abondants et plus diversifiés morphologiquement que chacun des autres groupes de parasites pris séparément. Cette grande variété dans 
les formes s'explique probablement par une plasticité particulière des Acariens, ellemême en rapport avec la taille très petite de ces arthropodes. Cette condition leur a permis de s'adapter à une gamme aussi variée d'hôtes et à occuper des niches parasitaires aussi extraordinaires et dont certaines sont uniques dans le monde parasitaire (Fain, 1975).

Certains groupes d'Acariens renferment à la fois des formes endoparasites, des ectoparasites et des formes libres. C'est le cas en particulier de certains Mésostigmates parasites d'oiseaux. Les voies respiratoires de la plupart des Ordres d'oiseaux hébergent des Mésostigmates parasites qui sont spécifiques pour cet habitat. Ils appartiennent tous à la famille Rhinonyssidae. On en connaît plusieurs centaines d'espèces. Ces Rhinonyssidae dérivent manifestement des Macronyssidae, qui vivent en ectoparasites sur divers Vertébrés, y compris les oiseaux. Les Macronyssidae eux-mêmes sont étroitement apparentés aux Laelapidae (Hypoaspidinae) libres vivant dans les nids de Vertébrés (Evans, 1955 ; Radovsky, 1967 et 1969).

Les Macronyssidae sont plus évolués que les formes libres dont ils dérivent, et les Rhinonyssidae sont à leur tour plus évolués que les Macronyssidae.

Ces Mésostigmates parasites d'oiseaux présentent donc un intérêt considérable, car ils forment une lignée phylogénétique complète sur le plan de l'adaptation parasitaire, et ils nous donnent l'occasion de suivre les diverses étapes par lesquelles sont passées les formes libres pour devenir finalement endoparasites.

Des lignées semblables plus ou moins complètes sont rencontrées encore dans d'autres groupes d'Acariens, comme les Prostigmates et les Astigmates.

Des circonstances aussi favorables pour l'étude du parasitisme n'existent chez aucun autre groupe de parasites, que se soient des Vers, des Insectes ou des Mallophages.

\section{Adaptations régressives et constructives \\ en rapport avec la vie parasitaire chez les Acariens}

L'étude de ces lignées d'Acariens, comprenant à la fois des formes libres, des ecto- et des endoparasites, nous a montré que deux ordres de phénomènes, indépendants l'un de l'autre, entrent en jeu dans l'adaptation au parasitisme. L'un est du type régressif, l'autre du type constructif (Fain, 1969).

Les phénomènes régressifs sont essentiellement caractérisés par une réduction ou la disparition de certains organes devenus inutiles. Le résultat final est une extrême simplification des différentes structures. Tous les organes peuvent être intéressés par ce processus. On assiste à la réduction ou à la disparition des écussons chitineux, des griffes des pattes, de la chaetotaxie, etc.

Indépendamment de ces phénomènes régressifs et en même temps que ceux-ci, on peut voir apparaître chez certains parasites, principalement les ectoparasites, des structures nouvelles. Celles-ci consistent essentiellement dans l'hypertrophie d'organes existants, plus rarement dans l'apparition d'organes nouveaux. Ces productions sont tou- 
jours des adaptations secondaires ou spécialisations destinées à répondre à une fonction nouvelle en rapport avec la vie parasitaire.

La fonction qui a induit les spécialisations les plus importantes est la fonction d'attache à l'hôte. C'est une fonction très importante, car c'est d'elle que dépend le maintien du parasite sur son hôte. C'est la fixation à la surface de la peau et aux poils qui a produit les structures les plus complexes et les plus puissantes. Certains de ces Acariens présentent un mélange de caractères les uns très régressés, les autres (organes d'attache) très spécialisés, ce qui montre que ces deux groupes de caractères relèvent de mécanismes différents.

Signalons encore que la régression n'intéresse pas toujours tous les organes de façon uniforme. Dans certains cas même, elle ne touche qu'un petit nombre de structures, laissant les autres non modifiées. Le parasite, dans ce cas, présente un mélange de caractères, les uns primitifs, les autres évolués. Il devient alors difficile de le situer exactement dans la lignée évolutive.

Les phénomènes de régression et, pour une moindre part, de spécialisation des organes, qui se manifestent lors du passage de la vie libre à la vie parasitaire, se retrouvent également au cours de l'évolution d'un parasite sur des hôtes de plus en plus évolués, comme cela a dû se passer au cours des périodes géologiques.

\section{Évolution comparée des parasites et de leur hôtes}

L'évolution a suivi dans son ensemble la voie de la complication des structures. Seuls les parasites font exception à cette règle et, chez eux, ce sont les phénomènes régressifs qui dominent le tableau ou sont les seuls représentés.

On ignore la cause de cette évolution à rebours chez les parasites, mais on peut présumer que les réactions immunitaires de défense de l'hôte y jouent un rôle important.

L'existence de réactions immunitaires de l'hôte est bien démontrée pour de nombreuses espèces d'ectoparasites producteurs de gale, ainsi que pour certains endoparasites. Il est probable que ces réactions existent à l'égard de tous les parasites, mais que, dans la plupart des cas, leur intensité reste trop faible pour être perçue cliniquement.

Les parasites disposent de divers moyens pour neutraliser les réactions de rejet de l'hôte. Certains, comme les Schistosomes, sont capables de se camoufler en s'entourant d'antigènes empruntés à leurs hôtes, ou encore en incorporant des protéines de l'hôte à leur propre substance. Un autre moyen pour un parasite de se faire tolérer par l'hôte serait de diminuer le plus possible la surface avec laquelle il entre en contact avec celui-ci, par exemple en réduisant la plupart de ses structures externes (poils, griffes, pattes, etc.).

Cette hypothèse suivant laquelle la régression des structures du parasite serait imposée par l'hôte et contrôlée grâce à la sélection naturelle rend bien compte des faits observés. En effet, c'est chez les hôtes les plus évolués, donc ceux qui présentent 
les systèmes immunitaires les plus perfectionnés, que l'on observe les parasites les plus régressés.

Un autre argument en faveur de cette hypothèse est fourni par l'étude des lignées phylogénétiques de Mésostigmates parasites d'oiseaux. Nous y voyons en effet que les Acariens qui vivent dans les voies respiratoires (Rhinonyssidae), et qui présentent donc un contact très intime avec les muqueuses de l'hôte, sont nettement plus régressés que ceux qui vivent sur la peau de ces mêmes hôtes (Macronyssidae), et qui n'ont donc avec ces derniers qu'un contact moins étroit.

On observe un phénomène similaire chez les vers du groupe des Nématodes, où les parasites tissulaires ou sanguicoles comme les Filaires sont plus évolués que les Nématodes vivant dans les cavités en communication avec le milieu extérieur.

Le mécanisme par lequel les réactions immunitaires de l'hôte influencent l'évolution de ses parasites est inconnu, mais c'est probablement en augmentant la pression de sélection sur le parasite, l'obligeant à sélectionner toujours la forme la plus régressée.

\section{Spécificité et évolution parallèle « hôtes-parasites 》 chez les Myobiidae}

\section{Spécificité chez les Myobiidae.}

Je voudrais montrer maintenant que les Acariens, à l'instar d'autres groupes de parasites, peuvent nous renseigner sur la parenté existant entre les hôtes sur lesquels ils vivent et aussi dans certains cas sur leur ancienneté relative.

Pour qu'un parasite puisse nous donner des indications valables dans ce domaine, il faut qu'il soit spécifique.

La spécificité des Acariens parasites est très variable suivant les groupes.

Les tiques (Métastigmates) et la plupart des Mésostigmates ectoparasitaires quittent fréquemment leur hôte, et à cette occasion peuvent passer sur un autre hôte. Leur spécificité est de ce fait généralement peu marquée.

Les plus spécifiques sont les parasites permanents, c'est-à-dire ceux qui passent toute leur existence sur leur hôte et ne le quittent à aucun moment.

Parmi ces parasites permanents, les Acariens pilicoles se caractérisent par une spécificité particulièrement stricte, qui s'explique probablement par la haute spécialisation des organes qui servent à la préhension du poil de l'hôte. Cette spécialisation, non seulement condamne l'Acarien définitivement à la vie pilicole, mais en outre elle établit une sélection parmi les hôtes, en éliminant ceux dont les poils ne s'adaptent pas à la pince pilicole.

La spécialisation pilicole semble donc particulièrement favorable au développement de la spécificité. On en trouve d'ailleurs un autre exemple chez les poux, qui sont également des parasites pilicoles et hautement spécifiques.

Les groupes d'Acariens pilicoles les plus importants font partie de la famille Myobiidae (Ordre des Prostigmates) et de la superfamille Listrophoroidea (Ordre des 
Astigmates). Je ne traiterai ici que des Myobiidae. Les Listrophoroidea feront l'objet d'un travail ultérieur.

La famille Myobiidae est divisée en trois sous-familles et compte 45 genres et 278 espèces. Ces Acariens vivent sur les Marsupiaux, les Insectivores, les Chauves-souris et les Rongeurs. Ce sont des pilicoles stricts qui s'attachent à leur hôte par la première paire de pattes fortement modifiées, et qui portent une sorte de pince dont les deux mors sont formés d'apophyses garnies de crêtes.

Chaque genre de Myobiidae est inféodé à un seul ordre d'hôtes.

Sauf quelques rares exceptions, tous les genres de Myobiidae restent cantonnés chacun dans une seule famille d'hôtes. La spécificité est particulièrement stricte chez les Myobiidae d'Insectivores, où chaque genre est inféodé à une sous-famille d'hôte, à l'exception toutefois d'un genre qui vit sur plusieurs sous-familles.

La spécificité sur le plan de l'espèce est très stricte. Dans la majorité des cas, une espèce de Myobiidae ne vit que sur un seul genre d'hôte.

Signalons encore que les hôtes primitifs hébergent habituellement des parasites primitifs. Il arrive cependant que des Myobiidae évolués soient rencontrés sur des hôtes primitifs. Ce cas se présente notamment pour les genres Microgale (Tenrecidae) et Elephantulus (Macroscelididae), qui hébergent des Myobiidae nettement plus évolués que ceux qui vivent sur la plupart des autres Insectivores. La présence de ces formes évoluées sur des hôtes primitifs résulte probablement d'une infestation secondaire de ces hôtes par des parasites en provenance d'hôtes plus évolués. Nous n'avons jamais observé le phénomène inverse, à savoir la présence d'un parasite primitif sur un hôte évolué. Cela semble indiquer qu'un hôte qui héberge un parasite primitif doit nécessairement être lui-même primitif. La raison de ce phénomène nous échappe, mais on peut supposer que le parasite primitif s'adapte moins facilement que le parasite évolué à un nouvel hôte, surtout lorsque ce nouvel hôte est plus évolué.

En raison de leur grande spécificité, les Myobiidae peuvent fournir des indications précieuses sur les affinités existant entre les hôtes.

\section{Evolution parallèle « hôtes-parasites » chez les Myobiidae.}

Les Myobiidae sont non seulement des parasites très spécifiques, mais divers arguments font penser qu'ils sont aussi des parasites très anciens, et que leur apparition pourrait remonter à l'époque de la formation des grands groupes de Mammifères. On peut donc présumer que hôtes et parasites ont évolué parallèlement au cours d'une longue période, ce qui laisse entrevoir la possibilité d'utiliser les Myobiidae comme des témoins de l'évolution des hôtes, et de préciser notamment l'ancienneté relative de certains genres ou familles de Mammifères (Fain, 1975).

Pour parvenir à ce but, il est indispensable de déterminer au préalable quels sont les critères d'ancienneté chez les Myobiidae. Une telle étude est difficile et elle suppose une connaissance approfondie de l'ensemble du groupe. 
Après avoir comparé et évalué tous les caractères morphologiques des Myobiidae, je suis arrivé à la conclusion que trois caractères sont particulièrement importants comme critères d'évolution, ce sont :

$1^{\circ}$ La structure de la première paire de pattes, c'est-à-dire celle qui sert à la fixation sur l'hôte.

$2^{\circ}$ Le nombre et le degré de réduction des griffes aux pattes.

$3^{\circ}$ La chaetotaxie.

Ces trois caractères sont de valeur très inégale.

Le plus important est la structure de la patte I. Ce caractère a évolué plus lentement que les autres, et il présente de ce fait une plus grande stabilité. Il permet de distinguer trois grands groupes parmi les Myobiidae.

1. Dans un premier groupe, tous les segments de la patte I sont libres et normalement développés et le tarse porte une paire de griffes, petites, mais normalement formées. Ce type de pattes est celui qui se rapproche le plus des formes ancestrales libres. On peut dire qu'il correspond aux espèces les plus primitives. Effectivement, ce groupe comprend toutes les espèces vivant sur les Marsupiaux américains ou australiens, soit 11 espèces réparties en 4 genres, et un genre monotypique parasitant un Insectivore asiatique. Tous ces hôtes sont primitifs.

2. Dans un deuxième groupe, la patte I présente une réduction plus ou moins marquée des segments apicaux avec, chez la plupart des espèces, une fusion plus ou moins complète du tarse avec le tibia. Les griffes terminales sont présentes ou absentes. Ce groupe est déjà plus évolué que le précédent. Il comprend tous les genres et espèces vivant sur les Insectivores (soit 14 genres et 53 espèces) excepté le genre monotypique cité plus haut, et toüs les genres et espèces (19 genres et 141 espèces) vivant sur les chauves-souris.

3. Enfin. dans le troisième groupe, la patte I est fortement raccourcie et présente une fusion complète des trois segments apicaux. Les griffes font toujours défaut. Ce groupe est représenté par 72 espèces réparties dans 6 genres. Il est complètement inféodé aux Rongeurs.

On constate donc qu'il existe une corrélation très nette entre le degré d'évolution de la patte I chez le parasite et la position systématique des hôtes.

\section{Marsupiaux}

Etudions maintenant la spécificité et l'évolution parallèle «hôte-parasite » dans les divers groupes d'hôtes.

Les Marsupiaux hébergent 11 espèces de Myobiidae, réparties dans 4 genres :

1. Le genre le plus primitif est Xenomyobia. Il est représenté par une seule espèce vivant sur Lestoros inca, un membre de l'archaïque famille Coenolestidae. 
C'est le Myobiidé le plus primitif connu jusqu'ici et probablement l'ancêtre de tout le groupe.

La première paire de pattes n'est pas modifiée. Tous les segments sont normalement développés, le tarse porte une paire de griffes, et il n'existe pas d'apophyses pilicoles. Cette patte ne peut donc pas servir à la préhension du poil de l'hôte. La fixation à l'hôte s'effectue au moyen des pattes III et IV qui sont terminées par des griffes très modifiées, très volumineuses, qui s'articulent avec des fortes épines situées sur les tibias.

2. Le deuxième genre connu chez les Marsupiaux est Archemyobia. Il comprend 7 espèces toutes inféodées aux Didelphidae américains. Chaque espèce est cantonnée dans un genre donné d'hôtes.

Dans ce genre, la première paire de pattes est du type primitif, c'est-à-dire avec tous les segments bien développés et une paire de griffes terminales. Cependant, contrairement au genre précédent, il existe un organe pilicole consistant en une gouttière chitineuse située sur la face interne du genu. Le poil de l'hôte est pincé entre ces deux gouttières et maintenu grâce au rapprochement des pattes I.

Les griffes III et IV ne sont pas modifiées et ne servent pas de pince pilicole.

3. Les deux autres genres connus chez les Marsupiaux sont Australomyobia et Acrobatobia. Ensemble, ils renferment 3 espèces vivant sur les Dasyuridae ou les Phalangeridae.

Le mode de fixation dans ces genres est différent de celui des deux genres précédents. Le poil de l'hôte est serré entre deux apophyses situées l'une sur le genu, l'autre sur le fémur de la patte I. Les griffes des pattes III et IV sont normales et ne servent pas à la fixation. Ce mode de fixation est identique à celui que l'on rencontre chez les Myobiidae plus évoluées vivant chez la majorité des Insectivores et la totalité des Chiroptères et des Rongeurs.

En résumé : on peut dire que les Marsupiaux de la famille Coenolestidae hébergent le genre de Myobiidae le plus primitif connu, qui est probablement l'ancêtre de tout le groupe.

Les Didelphidae hébergent un genre également très primitif, mais aberrant par la forme de son organe pilicole, qui est unique dans la famille Myobiidae.

Enfin, les Dasyuridae et Phalangeridae australiens hébergent deux genres plus évolués que les précédents, et dont l'organe pilicole a une structure semblable à celle des genres vivant sur la majorité des Insectivores et la totalité des Chiroptères et des Rongeurs.

\section{Insectivores}

\section{Spécificité chez les Myobiidae parasites des Insectivores.}

On a décrit chez les Insectivores 53 espèces et 15 genres de Myobiidae.

Tous ces genres et espèces sont strictement endémiques pour cet ordre d'hôtes. La spécificité est également très marquée à l'échelle de la famille ou même de la sous-famille de l'hôte. En effet, à l'exception du seul genre Eadiea, tous les genres de 
Myobiidae sont confinés dans une sous-famille d'Insectivores. A l'échelle de l'espèce, on constate également une étroite spécificité, chaque espèce étant pratiquement confinée dans un genre d'hôtes (Jameson, 1970 ; Fain et Lukoschus, 1976).

On peut conclure de ces observations que la spécificité des Myobiidae en provenance des Insectivores est particulièrement stricte.

\section{Evolution parallèle *hôtes-parasites " chez les Myobiidae parasites des Insectivores.}

Remarquons tout d'abord l'absence complète de Myobiidae chez les Chrysochloridae et les Tupaiidae, comme d'ailleurs chez les Primates, ce qui est peut-être un argument parasitologique pour séparer ces deux familles des autres familles d'Insectivores.

Nous voyons ensuite que les Insectivores hébergent deux genres de Myobiidae (Nectogalobia et Protomyobia) qui sont plus primitifs que tous les genres rencontrés chez les Chauves-souris. Ces deux genres vivent sur des Soricidae de la région holarctique. Tous les autres genres sont plus évolués. Ces constatations suggèrent que les Soricidae de la région holarctique constitueraient les groupes les plus primitifs des Insectivores, du moins de ceux dont on connaît des Myobiidae.

La corrélation entre l'évolution des Myobiidae et celle des Insectivores des familles Tenrecidae et Macroscelididae semble peu marquée si l'on juge d'après les caractères de la patte I, des griffes et de la chaetotaxie. Nous constatons en effet que le genre Elephantulobia qui vit sur un Macroscelididae et le genre Microgalobia qui parasite des Microgale spp. sont nettement plus évolués que la plupart des autres genres d'Insectivores. Cette situation paradoxale ne peut s'expliquer que si l'on admet que des Myobiidae sont passés secondairement d'hôtes évolués sur des hôtes plus primitifs. Ce passage s'est probablement effectué à plus d'une reprise au cours des périodes géologiques, et pas seulement chez les Insectivores. Des situations semblables existent en effet chez les Chiroptères (voir plus loin).

\section{Chiroptères}

Le troisième groupe d'hôtes des Myobiidae est celui des Chiroptères.

Les Chiroptères hébergent 141 espèces de Myobiidae réparties dans 20 genres, ce qui représente environ la moitié du nombre total de genres ou espèces connus de Myobiidae.

Les Microchiroptères interviennent dans ces totaux pour 131 espèces et 17 genres, les Mégachiroptères pour seulement 10 espèces et 3 genres.

La spécificité des Myobiidae de Chiroptères à l'échelle du genre est d'une façon générale bien marquée, mais cependant un peu moins stricte que chez les Insectivores.

Des trois genres vivant sur les Mégachiroptères, deux sont endémiques et un troisième est représenté également chez les Microchiroptères. Ce dernier genre est repré- 
senté par une espèce vivant chez Balionycteris (un Pteropidae), par une espèce vivant sur un Hipposideridae et par six espèces parasitant les Emballonuridae.

Au plan de l'espèce, la spécificité est très stricte, chaque espèce étant inféodée à un seul genre d'hôte. Il n'existe que quelques rares exceptions à cette règle, et peut-être s'agissait-il d'infestations accidentelles (Dusbabek, $1969 a$ et $1969 b$ ).

En ce qui concerne l'ancienneté des Myobiidae de Chiroptères, nous constatons que tous les genres vivant sur ces hôtes sont plus évolués que les trois genres vivant sur les Marsupiaux et que les genres les plus primitifs rencontrés sur les Insectivores. Cependant, certains genres vivant sur Microchiroptères (p. ex. Acanthophthirius) sont nettement plus primitifs que certains genres évolués vivant sur les Tenrecidae ou les Macroscelididae.

Nous voyons aussi que ce ne sont pas les Mégachiroptères qui hébergent les formes les plus primitives, mais les Microchiroptères, et en particulier les Vespertilionidae. En effet, les deux genres les plus primitifs connus chez les Microchiroptères sont Acanthophthirius et Pteracarus, représentés au total par 45 espèces toutes inféodées aux Vespertilionidae, à l'exception d'une espèce.

Ces constatations nous incitent à penser que les Myobiidae seraient passés des Insectivores sur les Chiroptères à une époque où la division de ces derniers en deux grands groupes était déjà réalisée. Les Vespertilionidae ont probablement été les premiers infestés, et c'est à partir de ces hôtes qu'ils seraient passés sur les autres groupes de Chiroptères.

Je pense qu'il est important de faire remarquer que ces considérations sur l'ancienneté du parasitisme chez les Chiroptères par les Myobiidae ne valent que pour ce groupe d'Acariens. Pour d'autres groupes d'Acariens, par exemple les Sarcoptidae et les Mésostigmates, ce sont les Mégachiroptères qui hébergent les formes les plus primitives et qui sont donc les hôtes les plus anciens.

\section{Rongeurs}

Le dernier ordre que nous allons étudier est celui des Rongeurs. Ceux-ci hébergent 73 espèces de Myobiidae groupées dans 6 genres.

\section{Spécificité des Myobiidae parasites des Rongeurs.}

Des 6 genres de Myobiidae connus chez les Rongeurs, 5 sont confinés chacun dans une famille de Rongeurs, le sixième (Radfordia) est divisé en 10 sous-genres qui vivent sur plusieurs familles de Rongeurs. Parmi ces sous-genres, il y en a trois qui mériteraient d'être érigés en genres distincts. Il s'agit de Cryptomyobia qui parasite les Bathyergidae, de Lavoimyobia qui vit sur les Heteromyidae et de Austromyobia qui vit sur les Gerbillidae, les Dipodidae et les Dendromyinae de la famille Muridae.

Il v a ensuite quatre sous-genres qui ne sont rencontrés que chez les Murinae. 
Enfin, il reste les trois sous-genres Radfordia, Graphiurobia et Microtimyobia. Le premier vit sur des Murinae et des Cricetinae ; le deuxième vit sur des Gliridae et des Zapodidae, et le troisième vit sur des Murinae, des Cricetinae et des Microtinae.

A l'échelle de l'espèce, la spécificité est stricte, chaque espèce étant confinée dans une sous-famille d'hôtes et dans la majorité des cas dans un genre d'hôtes (Fain, 1974).

\section{Evolution parallèle « hôtes-parasites »}

Nous constatons que les deux genres de Myobiidae les plus primitifs connus chez les Rongeurs ont été rencontrés l'un sur des Hystricomorphes néo-tropicaux (genre Proradfordia), l'autre sur un Anomaluridae africain (genre Idiurobia).

Le genre le plus évolué (Myobia), représenté par 12 espèces, n'est connu que des Murinae de l'Ancien Monde.

Les Gerbillidae sont parasités par cinq espèces du sous-genre Austromyobia (genre Radfordia) qui sont nettement plus primitives que les espèces vivant sur les Cricetidae et les Muridae, Murinae.

Les Rongeurs des genres Dendromus (Muridae: Dendromyinae) et Jaculus (Dipodidae) sont parasités par ce même sous-genre Austromyobia, ce qui permet d'entrevoir une relation entre les Gerbilles et les genres Dendromus et Jaculus.

Les Gliridae et les Zapodidae ne sont parasités que par le sous-genre Graphiurobia, ce qui suggère l'existence d'affinités entre ces deux familles. Cette hypothèse est renforcée par la découverte récente, chez ces deux familles, d'Acariens du genre Gliricoptes, appartenant à un tout autre groupe (Astigmata), et qui n'est représenté que chez ces hôtes.

Les Ctenodactylidae hébergent le genre Gundimyobia, qui présente un mélange de caractères, les uns évolués, les autres primitifs.

Les Muridae, Murinae et les Cricetidae sont parasités par les genres ou sousgenres les plus évolués de la famille.

Les Murinae hébergent les 12 espèces connues du genre Myobia, le plus évolué de la famille, ainsi que toutes les espèces des 4 sous-genres du genre Radfordia: Lophurmyobia, Hylomyscobia, Syconycterobia et Hystricomyobia. Trois de ces sous-genres forment des petits groupes isolés géographiquement. Les Murinae sont parasités, en outre, par un certain nombre d'espèces des sous-genres Microtimyobia et Radfordia, qui sont les plus évolués du genre. Des 22 espèces connues dans le sous-genre Radfordia, 15 vivent chez les Murinae, 5 chez les Cricetinae et 2 sur des hôtes accidentels. Les Murinae hébergent en outre 1 espèce du sous-genre Microtimyobia.

En ce qui concerne les Cricetidae, nous constatons que les Microtinae de l'Ancien et du Nouveau Monde hébergent uniquement des espèces (5 au total) du sous-genre Microtimyobia, alors que les Cricetinae hébergent 2 espèces de ce sous-genre (Ancien et Nouveau Monde) et 5 espèces du sous-genre Radfordia (Nouveau Monde).

Nous voyons donc que les Microtinae sont parasités seulement par le sous-genre Microtimyobia, alors que les Cricetinae et les Murinae hébergent des espèces des deux sous-genres Radfordia et Microtimyobia. 


\section{Bibliographie *}

DusbabeK (F.), $1969 a .-$ To the phylogeny of the genera of the family Myobiidae (Acarina). Acarologia, 11, 537-574.

DusbabeK (F.), $1969 \mathrm{~b}$. - To the phylogeny and zoogeography of bats (Chiroptera) based on the study of their parasitic mites (Acarina). Proc. First Intern. Bat Conference Czechoslovakia 6-9 Sept. 1968. Lynx. Mus. Nat. Praha, 10, 19-24.

FAIN (A.), 1969. - Adaptation to Parasitism in Mites. 2nd International Congress of Acarology in Sutton Bonington (England), 19-25 July 1967. Acarologia, 11, 429-449.

FAIN (A.), 1974. - Observations sur les Myobiidae parasites des Rongeurs. Evolution parallèle. Hôtes-Parasites (Acariens: Trombidiformes). Acarologia, 16, 441-475.

FAIN (A.), 1976. - Ancienneté et Spécificité des acariens parasites. Evolution parallèle. Hôtes-Parasites. Acarologia, 17, 369-374.

FAIN (A.), 1977. - Spécificité et Evolution parallèle Hôtes-Parasites chez les Acariens. I. Acariens pilicoles de la famille Myobiidae (Prostigmata) (sous presse).

Fain (A.) et Lukoschus (F.S.), $1976 a$. - Observations sur les Myobiidae d'Insectivores avec description de taxa nouveaux (Acarina: Prostigmates). Acta Zool. Path. Antverp, $\mathrm{n}^{\circ} 66$ : 121-188.

Fain (A.) et Lukoschus (F.S.), 1976 b. - A new genus and species of Myobiidae from the Marsupial Lestoros inca. Acarologia (sous presse).

Ellerman (J. R.), 1940. - The families and genera of living rodents, Brit. Mus. (Nat. Hist.), Vol. I (pp. 1-689) and II (pp. 1-690).

Evans (G. O.), 1955. - A review of the Laelaptid paraphages of the Myriapoda with descriptions of three new species (Acarina: Laelaptidae). Parasitology, 45, 352-368.

Grassé (P.-P.), 1955. - Traité de Zoologie. Tome XVII : Mammifères, fasc. 1 et 2, Masson, édit., Paris.

Gureev (A. A.), 1971. - Semleroiki fauny mira. Isd. Nauka., Leningrad, 253 p.

JAMESON (Jr E. W.), 1970. — Notes on some Myobiid mites (Acarina: Myobiidae) from Old World Insectivores (Mammalia: Soricidae and Talpidae). J. Med. Ent., 7, 79-84.

Lavocat (R.), 1973. - Histoire des Mammifères, $\mathrm{n}^{\circ}$ 28, pp. 1-190, Editions du Seuil, Paris.

Premier Symposium sur la Spécificité parasitaire des Parasites de Vertébrés 1957. Inst. Zool. Univers. Neuchâtel, 324 p.

RadovsKY (F. J.), 1967. - The Macronyssidae and Laelapidae (Acarina: Mesostigmata) parasitic on bats. Univ. Calif. Publ. Ent., 46, 1-288.

RAdOVSKY (F. J.), 1969. - Adaptative radiation in parasitic Mesostigmata. Symposia of the 2nd Intern. Congress of Acarology Sutton Bonington 1967. Acarologia, 11, 450-483.

* Une bibliographie plus complète est donnée dans le travail de Fain, 1977. 